\title{
A pseudoperceptual display system for investigating human statistical decision processes
}

\author{
G. LOWE and M. MARSH \\ University of Hull, Hull, England
}

A CRO display system is described in which a random sequence of dots appears on the CRO derived from a Gaussian distribution with a specified mean and standard deviation. Os are required to make judgments about the statistical parameters of the distributions.

Many recent studies of perceptual problems have utilized a normative decision model as a description of human perceptual decisions (e.g., Green \& Swets, 1966). However, there have been difficulties in measuring directly the efficiency of such perceptual judgments, since in most cases the statistical parameters of the stimulus situation cannot be measured exactly. The present paper describes a situation in which the relevant parameters can be specified precisely.

The experimental task involves judgments about parameters of a random Gaussian distribution of dots displayed sequentially on an oscilloscope screen along the vertical (Y) axis only. By this means, both "noise" or background (B) and signal (S) values are normally distributed, with the standard deviation of the distributions directly measurable. A signal consists of an upward deviation (or shift) of the dot distribution along the vertical axis. In addition, signal sample size is determined precisely by the number of observations in the sample or, in this case, the number of dots displayed sequentially on the CRO screen in a given sampling period. The O's task is to decide, on each trial, whether a given sample or sequence of dots came from the "noise"

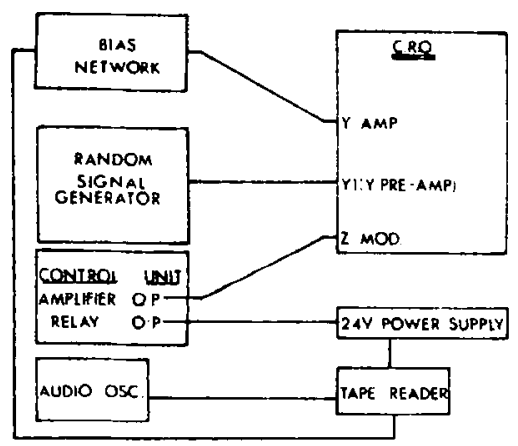

Fig. 1. Block schematic diagram of the CRO display system. a CRO screen. derived from a Gaussian distribution or from the deviated (signal) distribution.

In contrast with many typical perceptual situations, sensory transformations do not contribute directly to the magnitude of the "noise" involved. Investigations using the system are concerned directly with the actual decision processes involved in the perceptual task. Thus, ideal performance can be specified in all respects, enabling direct measures of efficiency to be obtained from a comparison of ideal and 0 performance.

The experimental setup involves a random sequence of dots appearing on distribution with a measurable mean and standard deviation. A shift in the mean level of this distribution during a specified temporal interval constitutes a signal.

Figure 1 shows the block schematic arrangement of the apparatus. An input (Y1) to the display oscilloscope from the random signal generator produces a random Gaussian waveform along the $\mathrm{Y}$-axis only when the $\mathrm{X}$ time

base is switched off (i.e., a one-dimensional display). The control unit incorporates a device for modulating the trace brightness and a variable-frequency multivibrator (MV). At each pulse from the MV only a single small illuminated dot can be seen on the screen at a position along the $\mathrm{Y}$-axis determined by the variable output from the random signal generator. Consequently, a discrete random sequence of dots appears on the CRO screen at a given frequency (i.e., a single dot appears sequentially at random positions up and down a single centralized vertical line-the Y-axis). Mean level and standard deviation are measured by a standard voltmeter and a rms meter. An observation interval or sequence on each stimulus trial is indicated by an equivalent sequence of auditory "pips" through a loudspeaker or earphones.

Signal "intensity" is determined by an upward shift of the mean level of the random noise distribution of dots along the $\mathrm{Y}$-axis. This is achieved by applying to the $\mathrm{Y}$ input a bias voltage, directly related to the mean value and standard deviation (or $\mathrm{rms} V$ ) of the distribution. Stimulus presentation, intensity values, and signal sample size are controlled by means of a programmed output from a tape reader, driven by an amplified pulse output from the MV. Alternative

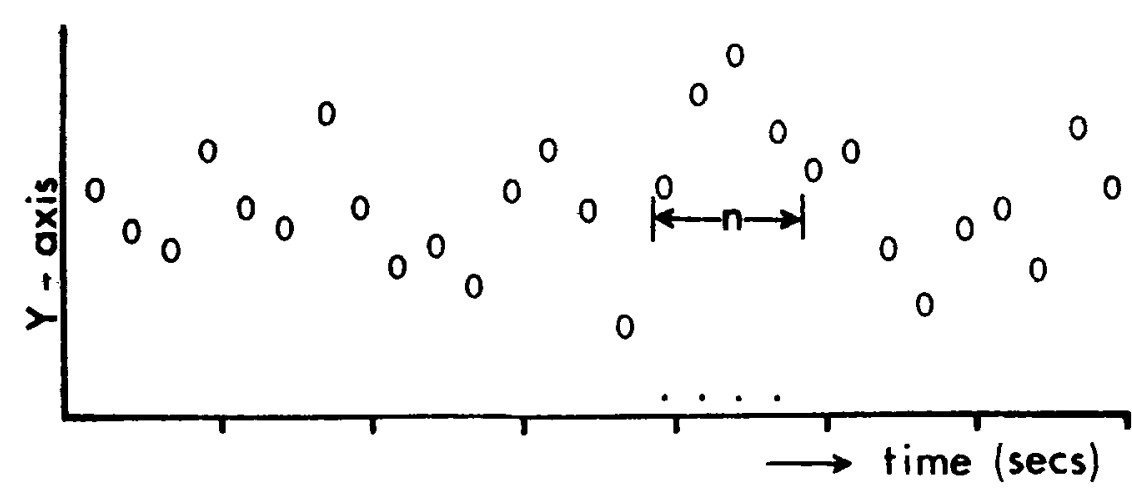

Fig. 2. Temporal representation of a typical CRO dot display, with a signal sample of four dots indicated by $n$ at a signal intensity of $1.0 \mathrm{SD}$. The ordinate represents the vertical position of the dot along the $\mathrm{Y}$-axis of the CRO screen. Dots indicate auditory pips defining the sample set. 


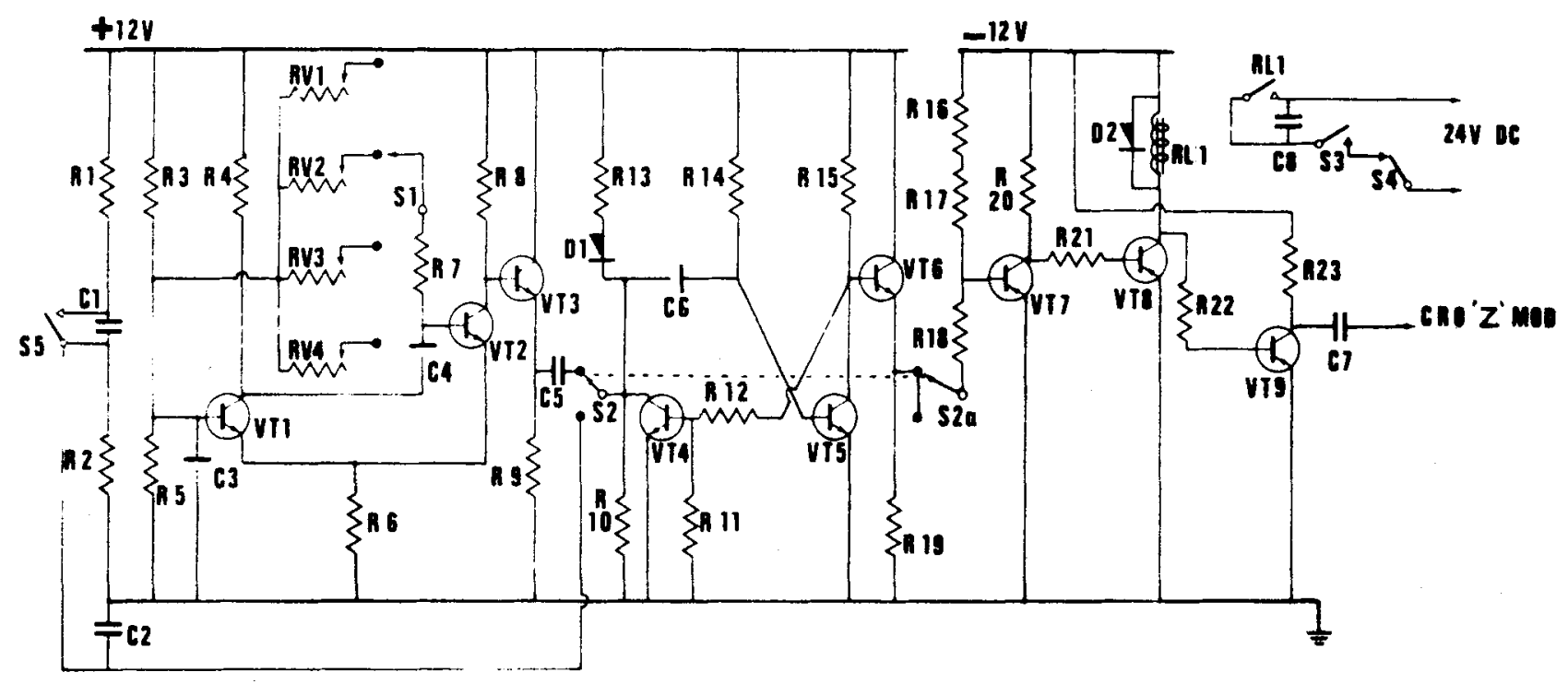

Fig. 3. Circuit diagram of the control unit.

Table 1

Component Designations for Control Unit

\begin{tabular}{|c|c|c|c|c|c|c|c|}
\hline $\mathbf{R 1}$ & $2.2 \mathrm{~K}$ & R13 & $10 K$ & C1. & $0.01 \mathrm{mfd}$ & VT 1 & BSY 95 \\
\hline $\mathbf{R 2}$ & $10 K$ & R14 & $100 K$ & $\mathrm{C2}$ & $0.003 \mathrm{mfd}$ & VT2 & BSY95 \\
\hline $\mathbf{R 3}$ & $6.8 \mathrm{~K}$ & R15 & $10 \mathrm{~K}$ & $\mathrm{C3}$ & $25 \mathrm{mfd}$ & VT3 & BSY95 \\
\hline R4 & $2.7 \mathrm{~K}$ & R16 & $11 \mathrm{~K}$ & $\mathrm{C} 4$ & $1.6 \mathrm{mfd}$ & VT 4 & BSY 95 \\
\hline $\mathbf{R 5}$ & 3.3K & R17 & $10 \mathrm{~K}$ & C5 & $0.001 \mathrm{mfd}$ & VT5 & BSY 95 \\
\hline R6 & $2.2 \mathrm{~K}$ & $\mathrm{R} 18$ & $1.6 \mathrm{~K}$ & C6 & $1.6 \mathrm{mfd}$ & VT6 & BSY 95 \\
\hline $\mathbf{R 7}$ & $1.6 \mathrm{~K}$ & R 19 & $1 \mathrm{~K}$ & C7 & $0.001 \mathrm{mfd}$ & VT7 & OC200 \\
\hline R8 & $2.7 \mathrm{~K}$ & R 20 & $10 \mathrm{~K}$ & $\mathrm{C8}$ & $0.1 \mathrm{mfd}$ & VT8 & OC84 \\
\hline $\mathbf{R 9}$ & $10 \mathrm{~K}$ & R21 & 47 oh & & & VT9 & OC84 \\
\hline R10 & 1 megohm & R22 & $6.2 \mathrm{~K}$ & & & & \\
\hline R11 & $15 \mathrm{~K}$ & R23 & $2.2 \mathrm{~K}$ & & & & \\
\hline R12 & $100 \mathrm{~K}$ & \multicolumn{6}{|c|}{ All resistors $0.125 \mathrm{~W}$} \\
\hline RV1 & 10K Preset & \multicolumn{6}{|c|}{ S1 course frequency control } \\
\hline RV2 & 100K Preset & \multicolumn{6}{|c|}{ S2, S2a free run or "one-shot" selection } \\
\hline RV3 & 220K Preset & \multicolumn{6}{|c|}{ S3 tape-reader on/off switch } \\
\hline RV4 & 470K Preset & \multicolumn{6}{|c|}{ S4 tape-reader contacts } \\
\hline D1 & OA200 & \multicolumn{6}{|c|}{ S5 push button for "one-shot" mode } \\
\hline D2 & OA5 & \multicolumn{6}{|c|}{$\mathrm{RL} / 112 \mathrm{~V}$ dc, 160 ohm coil } \\
\hline
\end{tabular}

systems of programmed switching may, of course, be used instead.

The CRO used in the present setup is an Airmec 279 display oscilloscope with a 17-in. tube. Its $\mathrm{Y}$ sensitivity range is linearly related to the voltage applied. The random-signal generator is a Servomex Type RG 77, which incorporates a thyratron source producing a flat noise spectrum over the frequency range of $30 \mathrm{~Hz}$ to $30 \mathrm{KHz}$. Its output is variable up to $5 \mathrm{~V} \mathrm{rms;} \mathrm{for} \mathrm{present} \mathrm{purposes} \mathrm{it} \mathrm{is}$ typically set at $1 \mathrm{~V}$ rms.

The control unit (see Fig. 3) consists of a free-running, emitter-coupled multivibrator (MV) with a bistable driving the output stage. This type of MV has excellent rise and fall time characteristics (Lewis, 1967), giving a good square wave at very low frequencies. In the present setup, the MV has switched resistive frequency components to give frequencies of $1,2,4$, and 8 pulses/sec; fine frequency control is obtained by preset miniature potentiometers. There is also a "one-shot" setting, allowing one pulse to pass whenever a manual pushbutton is depressed. This is used in "deferred decision" procedures, when Os are allowed to make as many observations as they need in order to reach a decision about the parameters of the sampled distribution (e.g., Swets \& Birdsall, 1967). The circuit utilizes Emitter Followers VT3 and VT6 as interstage buffers. The output of the bistable is fed into an amplifier (VT7 and VT8), which drives a $12-\mathrm{V}$ relay and a pulse output from VT9 via a capacitor (C7) to the $\mathrm{Z}$ modulation socket of the CRO. A capacitor value of $0.001 \mathrm{mF}$ was selected to produce a small well-focused spot on the CRO screen.

The bias network is used to control signal levels and contains a small $15-\mathrm{V}$ de power supply. This is applied via a bias on/off switch to a three-position rotary switch connected to potentiometers. Preset bias voltage levels, giving variable shifts along the $Y$-axis, can thus be switched in according to a given programmed sequence.

\section{REFERENCES}

GREEN, D. M \& SWETS J A Signal detection theory and psychophysics. New York: Wiley, 1966.

LEWIS, A. F. Constant voltage double pulse generators for use in electrophysiology. Electronic Engineering, 1967, 39, 470 .

SWETS, J. A., \& BIRDSALL, T. G. Deferred decisions in human signal detection: A preliminary experiment. Perception \& Psychophysics, 1967, 2 15-28. 